\title{
A GEOMETRICAL REGULARITY CRITERION IN TERMS OF VELOCITY PROFILES FOR THE 3D NAVIER-STOKES EQUATIONS
}

\author{
by C. V. TRAN and X. YU \\ (School of Mathematics and Statistics, University of St Andrews,
} St Andrews, KY16 9SS, United Kingdom)

(Department of Mathematical and Statistical Sciences, University of Alberta, Edmonton, AB, T6G 2G1, Canada)

[Received xx yy 2019. Revise xx yy 2019]

\section{Summary}

In this article we present a new kind of regularity criteria for the global well-posedness problem of the three-dimensional Navier-Stokes equations in the whole space. The novelty of the new results is that they involve only the profiles of the magnitude of the velocity. One particular consequence of our theorem is as follows. If for every fixed $t \in(0, T)$, the "large velocity" region $\Omega:=\left\{(x, t)|| u(x, t) \mid>C(q)\|u\|_{L^{3 q-6}}\right\}$, for some $C(q)$ appropriately defined, shrinks fast enough as $q \nearrow \varnothing$, then the solution remains regular beyond $T$. We examine and discuss velocity profiles satisfying our criterion. It remains to be seen whether these profiles are typical of general NavierStokes flows.

\section{Introduction}

The Navier-Stokes equations governing the motion of a viscous incompressible fluid are

$$
\begin{aligned}
& \frac{\partial u}{\partial t}+(u \cdot \nabla) u+\nabla p=\Delta u, \quad(x, t) \in \mathbb{R}^{3} \times(0, \infty) \\
& \nabla \cdot u=0, \quad(x, t) \in \mathbb{R}^{3} \times(0, \infty) \\
& u(x, 0)=u_{0}(x) \quad x \in \mathbb{R}^{3},
\end{aligned}
$$

where $u: \mathbb{R}^{3} \mapsto \mathbb{R}^{3}$ and $p: \mathbb{R}^{3} \mapsto \mathbb{R}$ are, respectively, the velocity and pressure fields and the viscosity is set to unity for convenience. Here $u_{0} \in L^{2}\left(\mathbb{R}^{3}\right)$ and $\nabla \cdot u_{0}=0$. The pioneering study on the Cauchy problem of this system by Leray (13) has established the existence of at least one solution (known as Leray-Hopf solution) $u \in L^{\infty}\left(0, T ; L^{2}\left(\mathbb{R}^{3}\right)\right) \cap L^{2}\left(0, T ; H^{1}\left(\mathbb{R}^{3}\right)\right.$ ) that takes the initial condition $u_{0}(x)$ in the $L^{2}$ sense and satisfies the energy inequality

$$
\|u\|_{L^{2}}^{2}+2 \int_{0}^{T}\|\nabla u\|_{L^{2}}^{2} \mathrm{~d} t \leq\left\|u_{0}\right\|_{L^{2}}^{2},
$$

for all $T>0$. Furthermore, if a Leray-Hopf solution becomes singular at some finite time $t=T_{*}$, then it is necessary that the velocity blows up in the following manner $(\mathbf{1 3})$

$$
\|u\|_{L^{s}} \geq \frac{c}{\left(T_{*}-t\right)^{(1-3 / s) / 2}}, \text { for } s>3 \text {, as } t \nearrow T_{*},
$$

Q. Jl Mech. Appl. Math. (2019) xx (x), 1-18

(C) Oxford University Press 2019 
where $c$ is a constant. It is easy to show that if a Leray-Hopf solution is smooth (regular), then it is unique and classical. Furthermore, given a sufficiently smooth $u_{0}$, such a solution has been known to remain smooth and unique up to some positive time $T_{0}$. The question is whether this localness can be removed, thereby extending $T_{0}$ to infinity for global regularity.

Decades of active research since Leray's seminal work has not been able to resolve the issue of global smoothness from first principles. To date, regularity has only been established under certain hypotheses. These hypotheses are known as regularity criteria, usually presupposing finiteness of quantities that are critical with respect to the invariant scalings $u(x, t) \mapsto \lambda u\left(\lambda x, \lambda^{2} t\right)$ and $p(x, t) \mapsto \lambda^{2} p\left(\lambda x, \lambda^{2} t\right)$, where $\lambda>0$ is a constant. An early result is the classical criterion by Prodi (14) and Serrin (15)

$$
\int_{0}^{T}\|u\|_{L^{s}}^{2 s /(s-3)} \mathrm{d} t<\infty, \text { for } s>3,
$$

which has direct bearing on the Leray singularity criterion (1.2). Apparently, the border case $s=3$ is not included in (1.3). Nonetheless, (1.3) itself suggests the criterion

$$
\operatorname{esssup}_{(0, T)}\|u\|_{L^{3}}<\infty \text {. }
$$

This turns out to be the case and criterion (1.4) was indeed proved by Escauriaza et al (6). Conditions (1.3) and (1.4) have been shown to belong to a large family of scaling invariant regularity criteria in Gibbon $(\mathbf{9})$, where the author further derived explicit gaps between such criteria and useful a priori bounds for weak solutions.

Criterion (1.3) remains valid when the norm $\|u\|_{L^{s}}$ is replaced by its scale-equivalent but marginally weaker counterpart $\|p\|_{L^{s / 2}}^{1 / 2}$. Indeed the criterion

$$
\int_{0}^{T}\|p\|_{L^{s}}^{2 s /(2 s-3)} \mathrm{d} t<\infty, \text { for } s>3 / 2,
$$

was proved by Chae and Lee (4) and Berselli and Galdi (1). However, unlike (1.4), it is not known whether $\|p\|_{L^{3 / 2}}<\infty$ would imply regularity.

Criteria (1.3) and (1.5) have been improved by a number of authors, primarily by replacing the $L^{s}$ norms by weaker but scale-equivalent norms or including a logarithmic factor. For example, Fan et al $(\mathbf{8})$ have replaced $\|p\|_{L^{s}}$ by the homogeneous Besov norm $\|p\|_{\dot{B}_{s, \sigma}^{0}}$ for some appropriate $\sigma$. Sohr (16) and Bosia et al (2) have used the weak Lebesgue norm $\|u\|_{L^{s, \infty}}$ in place of $\|u\|_{L^{s}}$. Zhou and Lei (22) have incorporated in (1.3) the factor $1 / \log \left(e+\|u\|_{L^{\infty}}\right)$. On the other hand, Fan et al (7) and Guo and Gala (11) have considered combinations of both types of these improvements. Recently, Tran and Yu (19) have derived a new type of improvement, weakening (1.3) by either the factor $1 /\left(1+\|u\|_{L^{3}}\right)^{\kappa}$ or $1 /\left(1+\|u\|_{\left.\dot{H}^{1 / 2}\right)^{\kappa}}\right.$ and $(1.5)$ by the factor $1 /\left(1+\|u\|_{L^{3}}\right)^{\kappa}$, for some suitable $\kappa>0$. There exist numerous results outwith the realm of Prodi-Serrin type criteria discussed presently. For example, Cao and Titi (3) and Zhou (21) have proved criteria expressible in terms of one component of the velocity field. Grujíc (10) has employed a geometrical approach, deriving criteria involving the geometry of regions of "intense fluid activity". Constantin and Fefferman (5) have employed another geometrical approach, establishing regularity constraints in terms of the direction of the vorticity.

This study features a new approach, also of geometrical nature, to the regularity problem 
of solutions of the Navier-Stokes equations. A regularity criterion is derived via new estimates for the pressure term in the equation governing the evolution of $\|u\|_{L^{q}}$, for $q \geq 3$. We examine high velocity region(s), denoted by $\Omega$, that is responsible for possible growth of $\|u\|_{L^{q}}$ and not known to be a priori controlled by viscous effects. Quantitatively, $\Omega$ is defined as follows. At each fixed time $t$, let

$$
U=U(t, q):=C(q)\|u\|_{L^{3 q-6}},
$$

where $C(q)$ will be specified in Lemma 2.2. In terms of $U, \Omega$ is given by

$$
\Omega=\Omega(t, q):=\{(x, t)|| u \mid>U\} .
$$

The pressure term is then estimated on the basis of possible flow structures within $\Omega$. The estimates are compared with the viscous dissipation term, whereby new results are deduced. A novelty of this study is that the results involve the velocity profile within $\Omega$ and its geometry. Another novelty is the application of pressure moderation, introduced in (18), that "cancels" the pressure term to some extent. Furthermore, a notable feature in this work is that estimates are carried out for different Lebesgue norms at each time $t$. In particular, there is no single $q$ such that the decay estimates for $\|u\|_{L^{q}}$ hold for all $t \in(0, T)$. Nevertheless, we show via a technical proposition (Proposition 2.4), which could be of interest by itself, that regularity can be guaranteed. We discuss the plausibility and implications of the derived criterion.

\section{Preliminaries}

\subsection{Pressure moderation}

The idea of pressure moderation, introduced in (18), is to apply an auxiliary function $\mathcal{P}$, called a pressure moderator, to minimize the nonlocal effect of the pressure term on the dynamics of $\|u\|_{L^{q}}$. For effective application of this idea, we need the following lemma, a refinement of Lemma 1 in (18).

Lemma 2.1. Let $u: \mathbb{R}^{3} \mapsto \mathbb{R}^{3}, f: \mathbb{R}^{3} \mapsto \mathbb{R}$, and $g: \mathbb{R}^{+} \cup\{0\} \mapsto \mathbb{R}$. Further assume that $\nabla \cdot u=0, u \cdot \nabla f=0, u \in H^{1} \cap L^{1} \cap L^{\infty}, f \in W^{1, \infty}$, and $g \in L^{\infty}$. Then for arbitrary $q \geqslant 0$, we have

$$
\int_{\mathbb{R}^{3}} \mathcal{P}|u|^{q} u \cdot \nabla|u| \mathrm{d} x=0
$$

where $\mathcal{P}(x):=f(x) g(|u|)$ is a pressure moderator.

Proof. Let

$$
H(s):=\int_{0}^{s} g(r) r^{q} \mathrm{~d} r .
$$

Then $H(s)$ is Lipschitz on the interval $\left[0,\|u\|_{L^{\infty}}+1\right]$. Furthermore we have $|H(s)| \leqslant$ $\|g\|_{L^{\infty}}|s|^{q+1} /(q+1)$, which, together with $u \in L^{1} \cap L^{\infty}$, implies $H(|u|) \in L^{2}$. Now by Theorem 2.1.11 of $(\mathbf{2 3})$ we have $H(|u|) \in H^{1}$ and

$$
u \cdot \nabla H(|u|)=g(|u|)|u|^{q} u \cdot \nabla|u|, \quad \text { a.e. }
$$

Multiplying both sides by $f(x)$ and integrating the resulting equation by parts, noting $u \cdot \nabla f=0$ and $\nabla \cdot u=0$, complete the proof. 


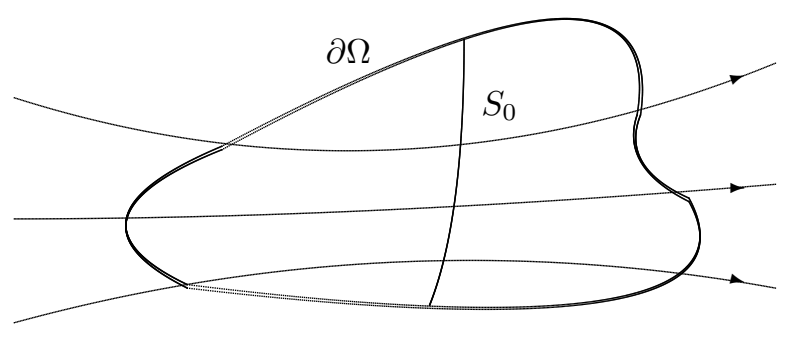

Fig. 1 A schematic description of the high velocity region $\Omega$, with boundary $\partial \Omega$. The directed curves represent streamlines. $S_{0} \subset \Omega$ is the surface where $u \cdot \nabla|u|=0$. The surface $S_{0}^{\prime}$ on which $u \cdot \nabla p=0$ (not shown) may lie relatively downstream or upstream compared with $S_{0}$. The former allows for fluid particles on $S_{0}$ to be accelerated and is the case of interest. The latter corresponds to fluid particles on $S_{0}$ being decelerated. The mixed possibility $S_{0} \cap S_{0}^{\prime} \neq \emptyset$ is also considered.

Throughout this study the pressure moderator $\mathcal{P}(x, t)$ is constructed as follows. Let $g(s)$ be defined by $g(s)=0$ for $s \in[0, U]$ and $g(s)=1$ for $s>U$, where $U$ is defined in (1.6). The choice of $f(x)$, which can be said to be a function of $t$ as well, depends on specific properties of the velocity field $u(x, t)$ and will be described in due course. It is worth mentioning that, following (1.7), $\mathcal{P}(x, t)=f(x, t) g(|u|)$ is supported on $\Omega$, independent of the form of $f(x, t)$.

\subsection{Basic energy estimates}

We assume a simple flow scenario, in which the boundary $\partial \Omega$ of the high velocity set $\Omega$ consists of finitely many closed surfaces that are sufficiently smooth to allow for the divergence theorem to hold. Note that despite the $C^{\infty}$ smoothness of $|u|$ for $t \in(0, T)$, no smoothness for the boundary of $\Omega(t, q):=\{(x, t)|| u \mid>U\}$ can be expected due to Whitney's extension theorem, which implies that any closed set can be the zero level set of a smooth function. Nevertheless, we may assume smoothness of $\partial \Omega$ through the following simple changes to our arguments. For every $(t, q)$ we pick $\tilde{U}(t, q) \in(U(t, q) / 2, U(t, q))$ such that $\tilde{U}(t, q)$ is a regular value of $|u|$. This is always possible due to Sard's theorem. We then define $\Omega(t, q)$ in terms of $\tilde{U}(t, q)$ instead of $U(t, q)$. It is easy to see that any compact subset of $\mathbb{R}^{3}$ can only contain finitely many connected components of $\Omega(t, q)$. Furthermore, thanks to the implicit function theorem, the boundary of each connected component of $\Omega(t, q)$ is smooth. A schematic description of one connected component of $\Omega$ is given by Figure 1. Now, it will be seen that all our arguments and conclusions still go through with this modified $\Omega(t, q)$.

Given the existence of a classical solution for $t \in(0, T)$, we may manipulate (1.1) in this time window of regularity without restrictions. The evolution of the local energy $|u|^{2} / 2$ is governed by

$$
\frac{\partial}{\partial t} \frac{|u|^{2}}{2}+u \cdot \nabla \frac{|u|^{2}}{2}+\nabla \cdot(p u)=\Delta \frac{|u|^{2}}{2}-|\nabla u|^{2} .
$$


Multiplying (2.2) by $|u|^{q-2}$ and integrating the resulting equation over $\mathbb{R}^{3}$ we obtain, after application of Lemma 2.1,

$$
\begin{aligned}
\frac{1}{q} \frac{\mathrm{d}}{\mathrm{d} t}\|u\|_{L^{q}}^{q}= & (q-2) \int_{\mathbb{R}^{3}} p|u|^{q-3} u \cdot \nabla|u| \mathrm{d} x \\
& -(q-2)\left\||u|^{(q-2) / 2} \nabla|u|\right\|_{L^{2}}^{2}-\left\||u|^{(q-2) / 2} \nabla u\right\|_{L^{2}}^{2} \\
= & (q-2) \int_{\mathbb{R}^{3}}(p+\mathcal{P})|u|^{q-3} u \cdot \nabla|u| \mathrm{d} x \\
& -(q-2)\left\||u|^{(q-2) / 2} \nabla|u|\right\|_{L^{2}}^{2}-\left\||u|^{(q-2) / 2} \nabla u\right\|_{L^{2}}^{2} \\
= & (q-2) \int_{\Omega}(p+f)|u|^{q-3} u \cdot \nabla|u| \mathrm{d} x \\
& +(q-2) \int_{\Omega^{c}} p|u|^{q-3} u \cdot \nabla|u| \mathrm{d} x \\
& -(q-2)\left\||u|^{(q-2) / 2} \nabla|u|\right\|_{L^{2}}^{2}-\left\||u|^{(q-2) / 2} \nabla u\right\|_{L^{2}}^{2}
\end{aligned}
$$

where $\Omega$ and $\mathcal{P}=f g$ are defined as in the final paragraph of the preceding subsection and $\Omega^{c}:=\mathbb{R}^{3} \backslash \Omega$. We treat the two integrals in (2.3) in turn.

On the one hand, since $|u|=U$ on $\partial \Omega$, integrating by parts the integral over $\Omega$ yields

$$
\begin{aligned}
\int_{\Omega}(p+f)|u|^{q-3} u \cdot \nabla|u| \mathrm{d} x & =\int_{\Omega}(p+f)|u|^{q-2} u \cdot \frac{\nabla(|u| / U)}{|u| / U} \mathrm{~d} x \\
& =\int_{\Omega} \log \frac{U}{|u|} \nabla \cdot\left((p+f)|u|^{q-2} u\right) \mathrm{d} x
\end{aligned}
$$

On the other hand, the integral over $\Omega^{c}$, with appropriate choice of $C(q)$, can be absorbed by the dissipation term as is seen from the following lemma.

LEMMA 2.2. For any $q \geq 8 / 3$, let $U(t, q)=C(q)\|u\|_{L^{3 q-6}}$ with $C(q)=$ $\left(c_{0} c_{1} q\left\|u_{0}\right\|_{L^{2}}\right)^{-2 /(q-2)}$. Here $c_{0}$ and $c_{1}$ are, respectively, the constants in the CalderónZygmund inequality

$$
\|p\|_{L^{2}} \leq c_{0}\|u\|_{L^{4}}^{2}
$$

and the Sobolev inequality

$$
\|h\|_{L^{6}} \leq c_{1}\|\nabla h\|_{L^{2}} .
$$

Further let $\Omega(t, q)=\{x|| u \mid>U(t, q)\}$. Then

$$
\int_{\Omega^{c}} p|u|^{q-3} u \cdot \nabla|u| \mathrm{d} x \leq \frac{1}{2}\left\||u|^{(q-2) / 2} \nabla|u|\right\|_{L^{2}}^{2} .
$$

Proof. Let $\widehat{u}:=u /|u|$. We have 


$$
\begin{aligned}
\int_{\Omega^{c}} p|u|^{q-3} u \cdot \nabla|u| \mathrm{d} x & \leq C^{(q-2) / 2}\|u\|_{L^{3 q-6}}^{(q-2) / 2}\|p\|_{L^{2}\left(\Omega^{c}\right)}\left\||u|^{(q-2) / 2} \widehat{u} \cdot \nabla|u|\right\|_{L^{2}\left(\Omega^{c}\right)} \\
& \leq C^{(q-2) / 2} c_{0}\|u\|_{L^{4}}^{2}\|u\|_{L^{3 q-6}}^{(q-2) / 2}\left\||u|^{(q-2) / 2} \widehat{u} \cdot \nabla|u|\right\|_{L^{2}\left(\Omega^{c}\right)} \\
& \leq C^{(q-2) / 2} c_{0}\|u\|_{L^{2}}\|u\|_{L^{3 q}}^{q / 2}\left\||u|^{(q-2) / 2} \widehat{u} \cdot \nabla|u|\right\|_{L^{2}\left(\Omega^{c}\right)}, \\
& \leq C^{(q-2) / 2} c_{0}\|u\|_{L^{2}}\|u\|_{L^{3 q}}^{q / 2}\left\||u|^{(q-2) / 2} \widehat{u} \cdot \nabla|u|\right\|_{L^{2}},
\end{aligned}
$$

where Calderón-Zygmund's inequality and the interpolation estimates

$$
\|u\|_{L^{4}} \leq\|u\|_{L^{2}}^{(3 q-4) /(6 q-4)}\|u\|_{L^{3 q}}^{3 q /(6 q-4)}
$$

and

$$
\|u\|_{L^{3 q-6}} \leq\|u\|_{L^{2}}^{4 /[(3 q-2)(q-2)]}\|u\|_{L^{3 q}}^{q(3 q-8) /[(3 q-2)(q-2)]}
$$

have been used. Here the latter estimate is valid for $q \geq 8 / 3$, although we are mainly concerned with $q \geq 3$. By applying Sobolev's inequality to $|u|^{q / 2}$ we obtain

$$
\|u\|_{L^{3 q}}^{q / 2} \leq \frac{c_{1} q}{2}\left\||u|^{(q-2) / 2} \nabla|u|\right\|_{L^{2}} .
$$

Substituting the above results into (2.8) yields

$$
\int_{\Omega^{c}} p|u|^{q-2} \widehat{u} \cdot \nabla|u| \mathrm{d} x \leq \frac{C^{(q-2) / 2} c_{0} c_{1} q\|u\|_{L^{2}}}{2}\left\||u|^{(q-2) / 2} \nabla|u|\right\|_{L^{2}}^{2}
$$

Finally, substituting $\|u\|_{L^{2}} \leq\left\|u_{0}\right\|_{L^{2}}$ and $C=\left(c_{0} c_{1} q\left\|u_{0}\right\|_{L^{2}}\right)^{-2 /(q-2)}$ proves the lemma.

Upon invoking Lemma 2.2 and (2.9), (2.3) reduces to

$$
\frac{\mathrm{d}}{\mathrm{d} t}\|u\|_{L^{q}}^{q} \leq q(q-2) \int_{\Omega} \log \frac{U}{|u|} \nabla \cdot\left((p+f)|u|^{q-2} u\right) \mathrm{d} x-\frac{2}{c_{1}^{2}}\|u\|_{L^{3 q}}^{q} .
$$

The reduced driving term in (2.10) is the subject of our present analysis.

REMARK 2.3. When $q=2,(2.3)$ reduces to

$$
\frac{1}{2} \frac{\mathrm{d}}{\mathrm{d} t}\|u\|_{L^{2}}^{2}=-\|\nabla u\|_{L^{2}}^{2}
$$

which readily implies the a priori estimate

$$
\int_{0}^{T}\|\nabla u\|_{L^{2}}^{2} \mathrm{~d} t \leq \frac{\left\|u_{0}\right\|_{L^{2}}^{2}}{2}
$$

In terms of scaling, the two bounded quantities in (2.11) are weaker than the scale-invariant quantities discussed earlier by a factor of $\lambda$. In the language of Navier-Stokes regularity theory, (1.1) is said to be supercritical with respect to the energy. It is folklore that this supercriticality can be removed and global regularity can be secured with the hyperviscosity term $-(-\Delta)^{5 / 4} u$ in place of the usual one (e.g. (12) and (17)). This necessary extra strength in dissipation totally agrees with the criteria discussed in the introductory section. 
The divergence $\nabla \cdot\left((p+\mathcal{P})|u|^{q-2} u\right)$ can be said to be symmetric, in the sense that it integrates over $\mathbb{R}^{3}$ to zero. In the driving term in $(2.3)$, which can be written as $(q-$ 2) $\int_{\mathbb{R}^{3}} \log (U /|u|) \nabla \cdot\left((p+\mathcal{P})|u|^{q-2} u\right) d x$, this symmetry is weakly broken by the logarithmic factor $\log (U /|u|)$. One may therefore expect that some cancellation occurs within the term. The question is to what extent such cancellation can be. The answer to this question depends on a number of factors. Firstly, the correlation between $|u|$ and $\nabla \cdot\left((p+\mathcal{P})|u|^{q-2} u\right)$, i.e. their relative spatial distribution, appears to be the key factor. Secondly, the divergence $\nabla \cdot\left((p+\mathcal{P})|u|^{q-2} u\right)$ itself depends on the correlation between $u$ and $p+\mathcal{P}$.

In the limit of large $q, C(q)$ tends to unity, and the driving set $\Omega$ reduces to point(s) of peak $|u|$. In passing, it is interesting to note that $\Omega$ as defined here is not optimal. More precisely, Tran and $\mathrm{Yu}(\mathbf{2 0})$ shows that $C(q)$ may be replaced by a time-dependent quantity, say $C(t, q)$, that grows and would blow up with $\|u\|_{L^{q}}$. The manner in which $C(t, q)$ grows with $\|u\|_{L^{q}}$ is not known in detail. Nevertheless, viscous effects can be stronger and $\Omega$ can be significantly smaller than presently considered. For simplicity, we use the present version of $\Omega$ with time-independent $C(q)$ throughout our analysis.

For the remainder of this study, we derive optimal estimates for the driving term in (2.10), i.e. the volume integral over $\Omega$, by elementary methods. We turn this volume integral into a surface integral, whenever possible, to avoid (presumably suboptimal) estimates involving the peak velocity in the interior of $\Omega$. The results are then compared with the dissipation term and regularity criteria are deduced.

\subsection{Regularity from instantaneous decay of Lebesgue norms}

The key argument in most regularity proofs is the establishment of the estimate $\mathrm{d}\|u\|_{L^{q}} / \mathrm{d} t \leqslant 0$ for some fixed $q \geqslant 3$ and all $t \in(0, T)$, from which regularity beyond $T$ easily follows through one of the Prodi-Serrin criteria. In contrast, the proof of our regularity criterion is more subtle, involving the following proposition, which essentially says decay of Lebesgue norms at every moment suffices. It should be emphasized that the decaying norms are allowed to be different at different moments.

Proposition 2.4. Let $u$ solve the $3 \mathrm{D}$ Navier-Stokes equations with initial value $u_{0} \in$ $C_{0, \sigma}^{\infty}\left(\mathbb{R}^{3}\right)$, the space of $C_{0}^{\infty}$ divergence free vector fields. Let $T>0$ be such that $u$ remains smooth on $(0, T)$. Assume that there is a positive function $Q(t) \in L_{l o c}^{\infty}([0, T))$ such that for every $t \in(0, T), \mathrm{d}\|u\|_{L^{q}} / \mathrm{d} t<0$ for all $q>Q(t)$. Then the solution remains smooth beyond $T$.

Proof. We give a proof by contradiction. Without loss of generality we assume $\left\|u_{0}\right\|_{L^{2}}<$ 1 , as otherwise we can consider $\lambda u_{0}(\lambda x)$ for appropriate $\lambda$. As $u_{0} \in C_{0, \sigma}^{\infty}\left(\mathbb{R}^{3}\right)$, we have $\left\|u_{0}\right\|_{L^{q}} \rightarrow\left\|u_{0}\right\|_{L^{\infty}}$ when $q \nearrow \infty$ and consequently there is $M>0$ such that

$$
\left\|u_{0}\right\|_{L^{q}} \leqslant M, \quad \forall q \geqslant 2
$$

Let $q_{0}>3$ be fixed. Recall that for the solution to blow up at $T$, there exists $c>0$ such that

$$
\|u(\cdot, t)\|_{L^{q_{0}}} \geqslant \frac{c}{(T-t)^{\left(1-3 / q_{0}\right) / 2}}
$$

It is clear that we can take $c<1$. 
Setting $\beta=\left(1-3 / q_{0}\right) / 4$ and $\delta^{\beta}=c$ yields

$$
\|u(\cdot, t)\|_{L^{q_{0}}} \geqslant \frac{1}{(T-t)^{\beta}}, \quad \forall t \in(T-\delta, T) .
$$

Now by interpolation we have

$$
\|u(\cdot, t)\|_{L^{q_{0}}} \leqslant\|u(\cdot, t)\|_{L^{2}}^{1-\alpha}\|u(\cdot, t)\|_{L^{q}}^{\alpha}
$$

where $\alpha=\frac{1 / 2-1 / q_{0}}{1 / 2-1 / q} \in(0,1)$, for all $q>q_{0}$. By $\left\|u_{0}\right\|_{L^{2}}<1$ and (2.14), we have

$$
\|u(\cdot, t)\|_{L^{q}} \geqslant \frac{1}{(T-t)^{\beta / \alpha}} \geqslant \frac{1}{(T-t)^{\beta}}, \quad \forall t \in(T-\delta, T) .
$$

Next we define $\bar{Q}(t):=\sup _{[0, t]} Q(\tau)$. Then clearly $\bar{Q} \in L_{l o c}^{\infty}(0, T), \bar{Q}(t) \geqslant Q(t)$ for all $t \in(0, T)$, and $\bar{Q}(t)$ is non-decreasing.

For any $t \in(0, T)$, we have

$$
q>\bar{Q}(t) \Rightarrow\|u(\cdot, t)\|_{L^{q}} \leqslant\left\|u_{0}\right\|_{L^{q}} \leqslant M .
$$

On the other hand, we have just shown that

$$
\|u(\cdot, t)\|_{L^{q}} \geqslant \frac{1}{(T-t)^{\beta}}, \quad \forall t \in(T-\delta, T) .
$$

Taking $t_{0} \in(T-\delta, T)$ such that $\frac{1}{\left(T-t_{0}\right)^{\beta}}>M$ and $q_{0}>\bar{Q}\left(t_{0}\right)$, we obtain contradiction.

\section{Main Results: instantaneous decay of Lebesgue norms}

We now present the main results of this study. Because of Proposition 2.4, for regularity, we only need to establish, at an arbitrary fixed time $t \in(0, T), \mathrm{d}\|u\|_{L^{q}} / \mathrm{d} t<0$ for all sufficiently large $q$. To handle the driving term in (2.10), we introduce some notation for convenience. First, let

$$
D(t, x, q):=\nabla \cdot\left((p+f)|u|^{q-2} u\right) .
$$

We then define $\Omega_{ \pm} \subset \Omega$ by

$$
\begin{aligned}
& \Omega_{+}(t, q):=\{x \in \Omega \mid D(t, x, q)<0\}, \\
& \Omega_{-}(t, q):=\{x \in \Omega \mid D(t, x, q)>0\} .
\end{aligned}
$$

As they stand, (3.1) and (3.2) mean that $\Omega_{+}$and $\Omega_{-}$make positive and negative contributions to the driving term in (2.10), respectively. For nontriviality, we obviously require positive measure of $\Omega_{+}$, i.e. $\left|\Omega_{+}\right|>0$. On the other hand, we consider both possibilities $\left|\Omega_{-}\right|=0$ (including $\Omega=\emptyset$ ) and $\left|\Omega_{-}\right|>0$. Remarkably, the former case turns out to be more favourable, although there is no partial cancellation of the driving term occurring within $\Omega$. For this case we have the following proposition.

Proposition 3.1. Let $\Omega_{ \pm}$be defined by (3.1) and (3.2). If $\left|\Omega_{-}\right|=0$ for all $q$ at some time $t \in(0, T)$, then $\mathrm{d}\|u\|_{L^{q}} / \mathrm{d} t<0$ at this time, for all large enough $q$. 
Proof. Since $D<0$ in $\Omega_{+}$, there exists a unique $U_{+} \in\left(U,\|u\|_{L^{\infty}}\right]$ such that

$$
\int_{\Omega_{+}} \log \frac{U}{|u|} D \mathrm{~d} x=\log \frac{U}{U_{+}} \int_{\Omega_{+}} D \mathrm{~d} x
$$

So

$$
\int_{\Omega} \log \frac{U}{|u|} D \mathrm{~d} x=\log \frac{U}{U_{+}} \int_{\Omega} D \mathrm{~d} x=U^{q-1} \log \frac{U}{U_{+}} \int_{\partial \Omega}(p+f) \widehat{u} \cdot \mathrm{d} a .
$$

Substituting this result with $U=C(q)\|u\|_{L^{3 q-6}}=\left(c_{0} c_{1} q\left\|u_{0}\right\|_{L^{2}}\right)^{-2 /(q-2)}\|u\|_{L^{3 q-6}}$ into (2.10) yields

$$
\begin{aligned}
\frac{\mathrm{d}}{\mathrm{d} t}\|u\|_{L^{q}}^{q} & \leq \frac{q(q-2)\|u\|_{L^{3 q-6}}^{q-1}}{\left(c_{0}^{2} c_{1}^{2} q^{2}\left\|u_{0}\right\|_{L^{2}}^{2}\right)^{(q-1) /(q-2)}} \log \frac{C\|u\|_{L^{3 q-6}}}{\|u\|_{L^{\infty}}} \int_{\partial \Omega}(p+f) \widehat{u} \cdot \mathrm{d} a-\frac{2}{c_{1}^{2}}\|u\|_{L^{3 q}}^{q} . \\
& =\left[\frac{q(q-2)}{\left(c_{0}^{2} c_{1}^{2} q^{2}\left\|u_{0}\right\|_{L^{2}}^{2}\right)^{(q-1) /(q-2)}} \log \frac{C(q)\|u\|_{L^{3 q-6}}}{\|u\|_{L^{\infty}}} \int_{\partial \Omega}(p+f) \widehat{u} \cdot \mathrm{d} a-\frac{2}{c_{1}^{2}} \frac{\|u\|_{L^{3 q}}^{q}}{\|u\|_{L^{3 q-6}}^{q-1}}\right]\|u\|_{L^{3 q-6}}^{q-1} .
\end{aligned}
$$

In the limit $q \nearrow \infty$ we have

$$
\begin{gathered}
\lim _{q \nearrow \infty} \frac{q(q-2)}{\left(c_{0}^{2} c_{1}^{2} q^{2}\left\|u_{0}\right\|_{L^{2}}^{2}\right)^{(q-1) /(q-2)}}=\frac{1}{c_{0}^{2} c_{1}^{2}\left\|u_{0}\right\|_{L^{2}}^{2}}, \\
\lim _{q \nearrow \infty} \log \frac{C(q)\|u\|_{L^{3 q-6}}}{\|u\|_{L^{\infty}}}=0
\end{gathered}
$$

and

$$
\begin{aligned}
\liminf _{q \nearrow \infty} \frac{\|u\|_{L^{3 q}}^{q}}{\|u\|_{L^{3 q-6}}^{q-1}} & \geq \liminf _{q \nearrow \infty}\|u\|_{L^{3 q}}\left(\frac{\|u\|_{L^{3 q}}}{\|u\|_{L^{2}}}\right)^{4(q-1) /[(3 q-2)(q-2)]} \\
& \geq \liminf _{q \nearrow \infty}\|u\|_{L^{3 q}}\left(\frac{\|u\|_{L^{3 q}}}{\left\|u_{0}\right\|_{L^{2}}}\right)^{4(q-1) /[(3 q-2)(q-2)]} \\
& =\|u\|_{L^{\infty}} .
\end{aligned}
$$

where the interpolation inequality $\|u\|_{L^{3 q-6}} \leq\|u\|_{L^{2}}^{4 /(3 q-2)(q-2)}\|u\|_{L^{3 q}}^{(3 q-8) q /(3 q-2)(q-2)}$ has been applied.

From the above limiting estimates, it is clear that $\mathrm{d}\|u\|_{L^{q}} / \mathrm{d} t<0$ for sufficiently large $q$ if the surface integral $\int_{\partial \Omega}(p+f) \widehat{u} \cdot \mathrm{d} a$ remains finite in the limit $q \rightarrow \infty$. This turns out to be the case. Indeed, we have

$$
\int_{\partial \Omega}(p+f) \widehat{u} \cdot \mathrm{d} a=\int_{\Omega} \nabla \cdot((p+f) \widehat{u}) \mathrm{d} x .
$$

Note that since $t \in(0, T)$ is fixed, the above integral will be uniformly bounded in $q$ as long as the volume of $\Omega(t, q)$ is so bounded.

Now by definition of $\Omega(t, q)$ we obtain

$$
|\Omega|<\frac{\|u\|_{L^{2}(\Omega)}^{2}}{C(q)^{2}\|u\|_{L^{3 q-6}}^{2}} \leq \frac{\left\|u_{0}\right\|_{L^{2}}^{2}}{C(q)^{2}\|u\|_{L^{3 q-6}}^{2}} \longrightarrow \frac{\left\|u_{0}\right\|_{L^{2}}^{2}}{\|u\|_{L^{\infty}}^{2}}
$$


as $q \nearrow \infty$. Consequently $|\Omega|<\infty$ uniformly for all large $q$ s. and the conclusion follows. The proof is thus completed.

REMARK 3.2. Intuitively, $|\Omega|$ vanishes in the limit $q \nearrow \infty$. This, together with the fact $\log (U /|u|) \rightarrow 0$ in $\Omega$, gives two vanishing factors in the driving term for $\|u\|_{L^{q}}$ in $(2.10)$. They compete with the growing factor $q(q-2)$. Proposition 3.1 shows that when $\left|\Omega_{-}\right|=0$, the vanishing terms dominate, leaving the pressure force overwhelmed by viscous effects. This is highly unexpected, and in stark contrast with the classical Prodi-Serrin type criteria, where the growth rate of some Lebesgue norm of $u$ has to be assumed.

When $\left|\Omega_{-}\right|>0$, there is some partial cancellation of the driving term in (2.10). This case appears more general than the earlier case $\left|\Omega_{-}\right|=0$. Let $U_{-} \in\left(U,\|u\|_{L^{\infty}}\right]$ be defined by

$$
\int_{\Omega_{-}} \log \frac{U}{|u|} D \mathrm{~d} x:=\log \frac{U}{U_{-}} \int_{\Omega_{-}} D \mathrm{~d} x .
$$

Similar to $U_{+}, U_{-}$is unique. The relative magnitudes of $U_{+}$and $U_{-}$and of $\int_{\Omega_{+}} D \mathrm{~d} x$ and $\int_{\Omega_{-}} D \mathrm{~d} x$ dictate the growth rate of $\|u\|_{L^{q}}$. With regard to $U_{+}$and $U_{-}$, there are two distinct cases to consider: $U_{-} \geq U_{+}$and $U_{-}<U_{+}$. Roughly speaking, the former case means that $D$ is more positive at higher $|u|$ and more negative at lower $|u|$. For the latter case, the distribution of $D$ is more negative at higher $|u|$ and more positive at lower $|u|$. We consider these cases in turn.

Proposition 3.3. Let $\Omega_{ \pm}$and $U_{+}$be defined as in Proposition 3.1. Let $U_{-}$be defined by (3.5). If $U_{-} \geq U_{+}$, then $\mathrm{d}\|u\|_{L^{q}} / \mathrm{d} t<0$, for all large enough $q$.

Proof. We have

$$
\begin{aligned}
\int_{\Omega} \log \frac{U}{|u|} D \mathrm{~d} x & =\log \frac{U}{U_{+}} \int_{\Omega_{+}} D \mathrm{~d} x+\log \frac{U}{U_{-}} \int_{\Omega_{-}} D \mathrm{~d} x \\
& \leq \log \frac{U}{U_{+}} \int_{\Omega_{+}} D \mathrm{~d} x+\log \frac{U}{U_{+}} \int_{\Omega_{-}} D \mathrm{~d} x \\
& =\log \frac{U}{U_{+}} \int_{\Omega} D \mathrm{~d} x
\end{aligned}
$$

From this point the proof proceeds in the same manner as the proof of Proposition 3.1.

Note that $U_{-} \geq U_{+}$is not necessarily indicative of substantial cancellation occurring between the positive and negative contributions. In fact, the level of cancellation can be wide-ranging. Negligible cancellation takes place if $\int_{\Omega_{-}} D \mathrm{~d} x \ll-\int_{\Omega_{+}} D \mathrm{~d} x$. On the other hand, substantial (or complete or over) cancellation occurs when $\int_{\Omega_{-}} D \mathrm{~d} x \approx-\int_{\Omega_{+}} D \mathrm{~d} x$.

Proposition 3.4. Let $\Omega_{ \pm}$and $U_{ \pm}$be defined as in Proposition 3.3. If $U_{-}<U_{+}$, then

$$
\begin{aligned}
\int_{\Omega} \log \frac{U}{|u|} D \mathrm{~d} x & =\log \frac{U_{-}}{U_{+}} \int_{\Omega_{+}} D \mathrm{~d} x+U^{q-1} \log \frac{U}{U_{-}} \int_{\partial \Omega}(p+f) \widehat{u} \cdot \mathrm{d} a \\
& =\log \frac{U_{+}}{U_{-}} \int_{\Omega_{-}} D \mathrm{~d} x+U^{q-1} \log \frac{U}{U_{+}} \int_{\partial \Omega}(p+f) \widehat{u} \cdot \mathrm{d} a .
\end{aligned}
$$


Proof. We have

$$
\begin{aligned}
\int_{\Omega} \log \frac{U}{|u|} D \mathrm{~d} x= & \log \frac{U}{U_{+}} \int_{\Omega_{+}} D \mathrm{~d} x+\log \frac{U}{U_{-}} \int_{\Omega_{-}} D \mathrm{~d} x \\
= & \log \frac{U}{U_{+}} \int_{\Omega_{+}} D \mathrm{~d} x+\log \frac{U_{-}}{U} \int_{\Omega_{+}} D \mathrm{~d} x \\
& +\log \frac{U}{U_{-}} \int_{\Omega_{+}} D \mathrm{~d} x+\log \frac{U}{U_{-}} \int_{\Omega_{-}} D \mathrm{~d} x \\
= & \log \frac{U_{-}}{U_{+}} \int_{\Omega_{+}} D \mathrm{~d} x+\log \frac{U}{U_{-}} \int_{\Omega} D \mathrm{~d} x
\end{aligned}
$$

Applying the divergence theorem to the second integral on the right-hand side, noting that $|u|=U$ on $\partial \Omega$, proves the first equation. The proof of the second equation is similar.

It is clear that if at some time $t \in(0, T), D<0$ in $\Omega$, then Proposition 3.1 applies and $\mathrm{d}\|u\|_{L^{q}} / \mathrm{d} t<0$ at this particular time. On the other hand, when $D>0$ almost everywhere in $\Omega$, the same conclusion trivially holds. This proves the following theorem.

TheOREM 3.5. Let $u$ solve the Navier-Stokes equations with $u_{0} \in C_{0, \sigma}^{\infty}\left(\mathbb{R}^{3}\right)$. Let $T>0$ be such that $u$ remains smooth up to (not including) time $T$. Assume that at every $t \in(0, T)$, there is a sequence of functions $f_{q}(x, t) \in W^{1, \infty}$, satisfying $u \cdot \nabla f_{q}=0$, such that $D=\nabla \cdot((p+$ $\left.\left.f_{q}\right)|u|^{q-2} u\right) \neq 0$ in $\Omega(t, q):=\{(x, t)|| u \mid>U(t, q)\}$ and $\lim _{\sup _{q}{ }^{\prime}}\left\|f_{q}(\cdot, t)\right\|_{W^{1, \infty}\left(\mathbb{R}^{3}\right)}<\infty$, then $u$ remains smooth beyond $T$.

REMARK 3.6. Theorem 3.5 involves ideas similar to those in (18), (20), and (10). On the other hand, no time regularity is required at all in Theorem 3.5, making it distinct from all previous regularity results. We present more discussions on this in Remarks 4.1 and 4.2.

The task now reduces to constructing a function $f$ that would render $D \neq 0$ in $\Omega$. In what follows we consider both possibilities that this condition holds and fails. For the former, the condition is satisfied by a simple form of $f$ and regularity is secured. For the latter, detailed analysis of proposition 3.4 yields a criterion that can be satisfied by reasonably realistic velocity profiles in $\Omega$.

\section{Discussions}

For the remainder of this study we apply Propositions 3.1, 3.3, 3.4, and in particular (3.6), to several possible flow structures in $\Omega$ and deduce the condition for regularity in each case. As seen from the proofs of these propositions, we may ignore the term corresponding to the integral over $\partial \Omega$ as this term is well controlled by the dissipation term.

By virtue of $\nabla \cdot u=0$ and $u \cdot \nabla f=0$, we have

$$
\begin{aligned}
D & =\nabla\left((p+f)|u|^{q-2}\right) \cdot u=|u|^{q-1} p^{\prime}+(q-2)|u|^{q-2}(p+f)|u|^{\prime} \\
& =\left(p^{\prime}+(q-2)(p+f) \frac{|u|^{\prime}}{|u|}\right)|u|^{q-1},
\end{aligned}
$$

where the symbol "'” denotes directional derivative along streamline. To determine whether 
$D$ vanishes in the interior of $\Omega$, we examine the solutions of

$$
p^{\prime}+(q-2)(p+f) \frac{|u|^{\prime}}{|u|}=0,
$$

or in a more compact form,

$$
(\log |p+f|)^{\prime}+(q-2)(\log |u|)^{\prime}=0 .
$$

Let $S_{0}^{\prime}:=\left\{(x, t) \mid p^{\prime}=0\right\}$. Recall that $S_{0}=\left\{\left.(x, t)|| u\right|^{\prime}=0\right\}$ (cf. Figure 1). For smooth flows, it is reasonable to assume that $S_{0}^{\prime}$ and $S_{0}$ are surfaces, consisting, respectively, of minima of $p$ and maxima of $|u|$ along the streamlines in $\Omega$. Here, we exclude minima of $|u|$ and maxima of $p$ for the reason that the high velocity set $\Omega$ is small enough so that each streamline within $\Omega$ does not have multiple extrema of each $|u|$ and $p$. Assume for the moment that $S_{0}^{\prime} \cap S_{0}=\emptyset$. There are two distinct cases to consider. $S_{0}^{\prime}$ is situated upstream (i) and downstream (ii) relative to $S_{0}$. On a given streamline $\ell$ passing through $\Omega$, let $O$ and $O^{\prime}$ be the points where $|u|^{\prime}=0$ and $p^{\prime}=0$, respectively, i.e. $O=\ell \cap S_{0}$ and $O^{\prime}=\ell \cap S_{0}^{\prime}$. Now define $f$ by $p+f=0$ on $S_{0}$ and $u \cdot \nabla f=0$. Note that as $p$ and $u$ are smooth and bounded, $f$ belongs to $W^{1, \infty}$. With respect to $O,|u|^{\prime}$ is positive and negative upstream and downstream, respectively. With respect to $O^{\prime}, p^{\prime}$ is negative and positive upstream and downstream, respectively.

For case (i), the signs of all quantities involved in the determination of the zeros of $D$ along $\ell$ are given by figure 2. It can be seen that relative to $O, p+f<0$ upstream and $p+f>0$ downstream. On the contrary, $|u|^{\prime}>0$ upstream and $|u|^{\prime}<0$ downstream. It follows that the product $(p+f)|u|^{\prime}$ is negative in the vicinity of $O$ (vanishing at $O$ though). Meanwhile $p^{\prime}>0$ in that region. As a result, $D$ vanishes at 2 points near $O$, one on the left, say $A$, and one on the right, say $B$. Note that $A$ and $B$ are closer to $O$ for larger $q$. Furthermore, $D>0$ between $A$ and $B$ and $D<0$ otherwise. It follows that $\Omega_{-}$and $\Omega_{+}$ comprise the inner and outer parts of $\Omega$, respectively. Hence $U_{-}>U_{+}$and proposition 3.3 applies to this case. Regularity thus follows. This makes sense as highest velocity fluid in the vicinity of $S$ decelerates in this case.

Similar to figure 2, figure 3 describes the flow data of interest for case (ii). As in case (i), $D$ vanishes at 2 points $A$ and $B$ on the left and right of $O$, respectively. However, the signs of $D$ on $\ell$ are opposite to those in case (i). Namely, $D<0$ between $A$ and $B$ and $D>0$ otherwise. This means that $\Omega_{+}$and $\Omega_{-}$comprise the inner and outer parts of $\Omega$, respectively. Hence $U_{+}>U_{-}$and proposition 3.4 applies to this case. Figure 4 describes the sets $\Omega_{-}$and $\Omega_{+}$separated by surfaces $S$ and $S^{\prime}$ on which $D=0$.

When $q$ increases, $\Omega$ shrinks and $A$ and $B$ approach $O$. If the shrinking rate is greater than the approaching rate, then eventually $A$ and $B$ will be left outside $\Omega$. This means that $D<0$ in $\Omega$, so Theorem 3.5 holds and regularity is secured. To see the plausibility of this scenario, we resort to the following rough estimate.

Let $d(\Omega)$ be the arc length of the longest streamlines within $\Omega$. On $S_{0}$ we have $p+f=0=$ $|u|^{\prime} /|u|$. By the Mean Value Theorem we can see that, for large $q,\left.|(q-2)(p+f)| u\right|^{\prime} /|u| \mid \leq$ $c(q-2) d(\Omega)^{2}$ in $\Omega$ for some constant $c$. On the other hand, Since $\min _{S_{0}}\left|p^{\prime}\right|>0$ we have $\left|p^{\prime}\right| \geq C$ for some positive constant $C$. Consequently, if $d(\Omega)=o\left(q^{-1 / 2}\right)$, eventually $D<0$ in $\Omega$ and regularity is secured. 


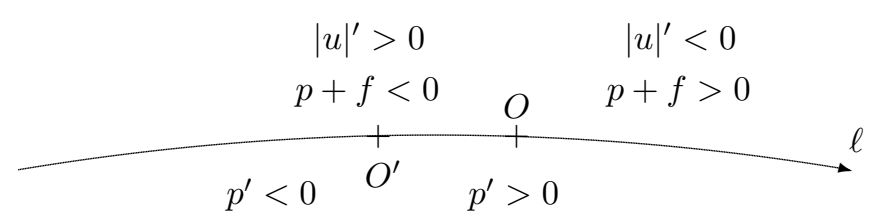

Fig. 2 The signs of various quantities determining the signs of $D$ along a streamline $\ell$ for case (i) in which $O^{\prime}$ (where $p^{\prime}=0$ ) is relatively upstream compared with $O$ (where $|u|^{\prime}=0$ ). The intersections of $\ell$ and $\Omega$ are omitted. For large enough $q, D=\left(p^{\prime}+(q-2)(p+f)|u|^{\prime} /|u|\right)|u|^{q-1}$ vanishes at 2 points near $O$ ( $A$ on the left and $B$ on the right - not shown). Furthermore, $D>0$ between $A$ and $B$ and $D<0$ otherwise.

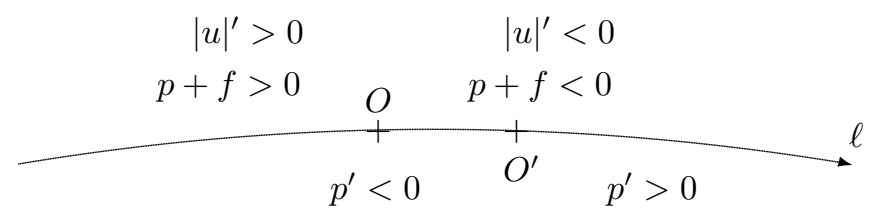

Fig. 3 The signs of various quantities determining the positions of the zeros of $D$ along a streamline $\ell$ for case (ii) in which $O^{\prime}$ is relatively downstream compared with $O$. Similar to case (i), $D=0$ at 2 points $A$ and $B$ on the left and right of $O$, respectively. But here $D<0$ between $A$ and $B$ and $D>0$ otherwise.

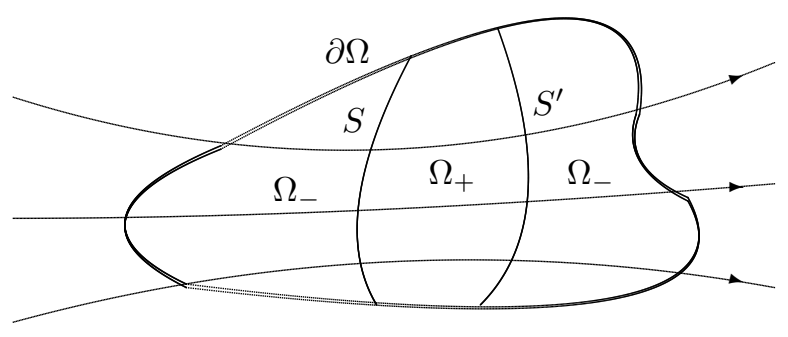

Fig. 4 A sketch of the sets $\Omega_{ \pm}$and their separating surfaces $S$ and $S^{\prime}$, where $D=0$, for case (ii) in which $S_{0}^{\prime}$ lies relatively downstream compared with $S_{0}$. The sets $\Omega_{+}$and $\Omega_{-}$comprise the inner and outer parts of $\Omega$, respectively. For case (i), these sets swap their positions. 
REMARK 4.1. Although the same argument can be applied when the pressure moderator is absent, that is $f=0$, the resulting criterion, $d(\Omega)=o\left(q^{-1}\right)$, is a much weaker result.

REMARK 4.2. The above discussion shows that when the high velocity region $\Omega(t, q)$ shrinks fast enough at every fixed time $t$ as $q \nearrow \infty$, then the solution remains regular. This is in spirit similar to the main result in Grujic (10), where the limiting behaviour of $\Omega_{s}(M(t)):=$ $\left\{x \in \mathbb{R}^{3}|| u(x, s) \mid>M(t)\right\}$ is involved. Here $M(t)$ is defined through $\|u\|_{L^{\infty}}$ and $s \in$ $(t, T)$ is some later time, with $T$ being the alleged blow-up time. On the other hand, we notice that the definitions of the high velocity regions $\Omega(t, q)$ and $\Omega_{s}(M)$ are very different. Furthermore, in (10) the limit involved is that of the time $t \nearrow T$, while in our discussion the time $t$ is fixed.

When $\Omega$ does not shrink fast enough, $A$ and $B$ remain in the interior of $\Omega$ for all $q$, and we can only apply Proposition 3.4. In this case, further analysis of either (3.6) or (3.7) is necessary to determine whether or not $\|u\|_{L^{q}}$ decays. We analyse the former in what follows.

From Propositions 3.1 and 3.3 we know that the surface integral over $\partial \Omega$ in (3.6) can be fully controlled by the dissipation term. So we ignore this integral and are mainly concerned with the integral $\int_{\Omega_{+}} D \mathrm{~d} x$, to which the divergence theorem is assumed to apply. With this assumption we have

$$
\int_{\Omega_{+}} D \mathrm{~d} x=\int_{\partial \Omega_{+}}(p+f)|u|^{q-1} \widehat{u} \cdot \mathrm{d} a .
$$

Now $\partial \Omega_{+}$consists of surfaces $S$ and $S^{\prime}$, on which $D=0$, and portions from $\partial \Omega$ (see figure 4). By the reason discussed above, the contributions from these portions may be ignored. Hence we may write

$$
\begin{aligned}
\int_{\Omega_{+}} D \mathrm{~d} x & \sim \int_{S \cup S^{\prime}}(p+f)|u|^{q-1} \widehat{u} \cdot \mathrm{d} a \\
& =\frac{1}{2-q} \int_{S \cup S^{\prime}} \frac{|u|}{|u|^{\prime}} p^{\prime}|u|^{q-1} \widehat{u} \cdot \mathrm{d} a .
\end{aligned}
$$

where (4.2) has been used. It follows that

$$
\begin{aligned}
\int_{\Omega} \log \frac{U}{|u|} D \mathrm{~d} x & \sim \log \frac{U_{-}}{U_{+}} \frac{1}{2-q} \int_{S \cup S^{\prime}} \frac{|u|}{|u|^{\prime}} p^{\prime}|u|^{q-1} \widehat{u} \cdot \mathrm{d} a \\
& \leq \log \frac{U_{-}}{U_{+}} \frac{\|u\|_{L^{\infty}\left(S \cup S^{\prime}\right)}^{q-1}}{2-q} \int_{S \cup S^{\prime}} \frac{|u|}{|u|^{\prime}} p^{\prime} \widehat{u} \cdot \mathrm{d} a .
\end{aligned}
$$

Comparing this estimate with the viscous dissipation term yields the following criterion. If

$$
\log \frac{U_{+}}{U_{-}} \int_{S \cup S^{\prime}} \frac{|u|}{|u|^{\prime}} p^{\prime} \widehat{u} \cdot \mathrm{d} a \leq \frac{2\|u\|_{L^{3 q}}^{q}}{c_{1}^{2} q\|u\|_{L^{\infty}\left(S \cup S^{\prime}\right)}^{q-1}},
$$

then $\mathrm{d}\|u\|_{L^{q}} / \mathrm{d} t<0$.

Since $U_{ \pm} \in\left(U,\|u\|_{L^{\infty}}\right)$, the prefactor $\log \left(U_{+} / U_{-}\right)$in (4.7) can become small in the limit 


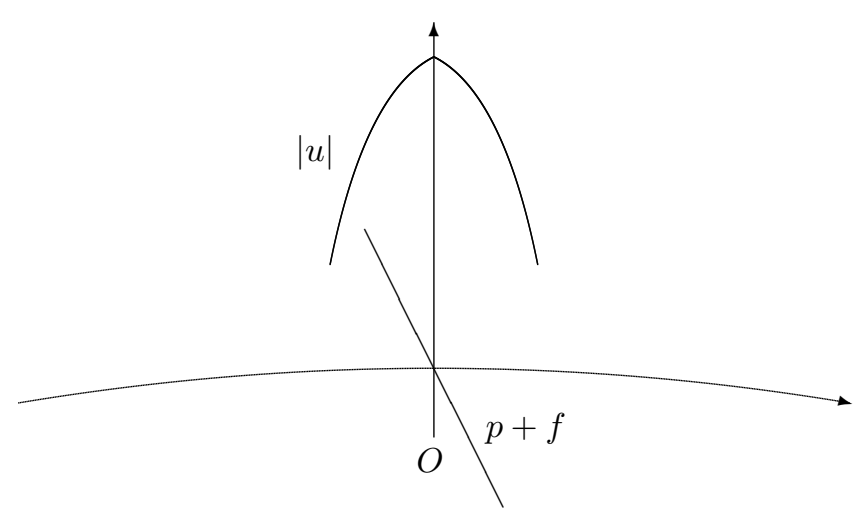

Fig. 5 Typical shapes of $p+f$ and $|u|$ near peak velocity at $O$, where $p+f=0=|u|^{\prime}$ and $p^{\prime}<0$.

$U \rightarrow\|u\|_{L^{\infty}}$, which is certainly achievable as $q \rightarrow \infty$. To have a sense of how small $\log \left(U_{+} / U_{-}\right)$can become, let $|u|$ scale as $|\Omega|^{-\alpha}$. Here one may allow $\alpha>1 / 3$ to include the unfavourable possibility $\|u\|_{L^{3}} \rightarrow \infty$ as $|\Omega| \rightarrow 0$. Let $U_{ \pm}=\|u\|_{L^{q_{ \pm}}}$, we then have $U_{ \pm} \sim|\Omega|^{-\alpha+1 / q_{ \pm}}$. It follows that $U_{+} / U_{-} \sim|\Omega|^{\left(q_{-}-q_{+}\right) / q_{-} q_{+}}$and

$$
\log \frac{U_{+}}{U_{-}} \sim \frac{q_{-}-q_{+}}{q_{-} q_{+}} \log |\Omega| .
$$

Now since $q_{+}>q_{-} \sim 3 q$, the ratio $\left(q_{+}-q_{-}\right) /\left(q_{-} q_{+}\right)$can be as small as $1 / q$ or smaller. This means that the logarithmic factor on the left-hand side of (4.7) may cancel the factor $1 / q$ on the right-hand side. On the other hand, the same scaling $|u| \sim|\Omega|^{-\alpha}$ implies that

$$
\frac{\|u\|_{L^{3 q}}^{q}}{\|u\|_{L^{\infty}\left(S \cup S^{\prime}\right)}^{q-1}} \geqslant \frac{\|u\|_{L^{3 q}}^{q}}{\|u\|_{L^{\infty}}^{q-1}} \sim|\Omega|^{1 / 3-\alpha} .
$$

Hence, (4.7) holds if $\int_{S \cup S^{\prime}} p^{\prime}|u| /|u|^{\prime} \widehat{u} \cdot \mathrm{d} a$ grows less rapidly than $|\Omega|^{-\beta}$ for some $0<\beta<$ $\alpha-1 / 3$.

The profile of $|u|$ near $O$ and the two-dimensional measure of $S$ and $S^{\prime}$ play key roles in the magnitude of the integral $\int_{S \cup S^{\prime}} p^{\prime}|u| /|u|^{\prime} \widehat{u} \cdot \mathrm{d} a$. Typical shapes of $p+f$ and $|u|$ near $O$ are given in figure 5 for illustration and are consistent with the assumption that $p^{\prime}$ does not change dramatically near $O$ while $|u|^{\prime}$ tends to zero in some manner as $A$ and $B$ approach $O\left(S\right.$ and $S^{\prime}$ approach $\left.S_{0}\right)$. Observe that the ratio $|u| /|u|^{\prime}$ is sensitive to the shape of $|u|$ but virtually independent of its magnitude. Let $s$ denote the arc length along $\ell$ with $s=0$ at $O$. Consider $|u(s)|=|u(0)| \theta(s)$, for some shape function $\theta(s)$ such that $\theta(0)=1$ and $\theta^{\prime}(0)=0$. This allows us to write

$$
\int_{S \cup S^{\prime}} \frac{|u|}{|u|^{\prime}} \widehat{u} \cdot \mathrm{d} a=\int_{S \cup S^{\prime}} \frac{1}{\theta^{\prime}} \widehat{u} \cdot \mathrm{d} a .
$$




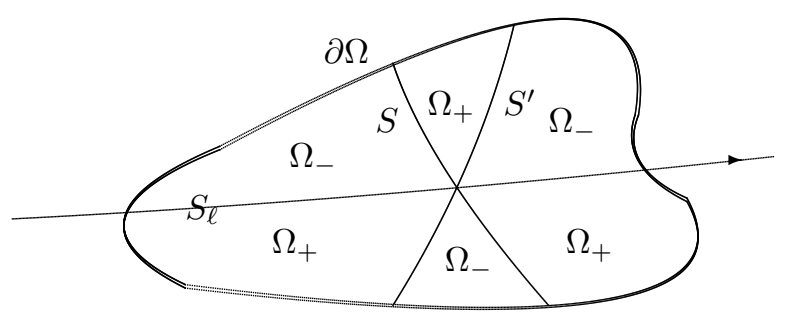

Fig. 6 A sketch of the sets $\Omega_{ \pm}$when $S_{0}$ and $S_{0}^{\prime}$ cross each other. Each $\Omega_{-}$and $\Omega_{+}$comprises three pieces, separated by $S, S^{\prime}$ and $S_{\ell}$. Continuity requires $D=0$ on $S_{\ell}$.

As $q \rightarrow \infty, S$ and $S^{\prime}$ collapse onto $S_{0}$. Hence in this limit we have $|S| \sim\left|S^{\prime}\right| \sim\left|S_{0}\right| \approx|\Omega|^{2 / 3}$. The above integral diminishes and (4.7) holds if, on $S$ and $S^{\prime}, \theta^{\prime}(s)$ tends to zero no more rapidly than $|\Omega|^{2 / 3+\beta}$ for some $\beta<\alpha-1 / 3$. A broad class of $\theta(s)$ satisfies this condition. A simple but reasonably realistic example is $\theta(s)=\cos \left(\eta|s|^{\gamma}\right)$, where $\eta>0$ and $\gamma \geq 1 / 2$. For this form of $\theta$, the sharpness of the velocity peak can be increased by either increasing $\eta$ or decreasing $\gamma$, or both.

Finally, when $S_{0} \cap S_{0}^{\prime} \neq \emptyset$, complications arise in the geometry of $\Omega_{ \pm}$, requiring minor modifications to the above analysis. If $S_{0}$ and $S_{0}^{\prime}$ just touch each other without crossing, then we have either case (i) or case (2), depending on whether $S_{0}^{\prime}$ lies relatively upstream or downstream compared with $S_{0}$. So the above analysis applies to this case without change. When $S_{0}$ and $S_{0}^{\prime}$ cross each other, $\Omega$ can be divided into 2 parts. These parts are separated by a surface, say $S_{\ell}$, which is made up of streamlines intersecting $S_{0} \cap S_{0}^{\prime}$. In one part, figure 2 applies to its streamlines. On the other hand, figure 3 applies to streamlines in the other part. For a streamline on $S_{\ell}, D$ vanishes on the entire line. Hence $D=0$ on $S, S^{\prime}$ and $S_{\ell}$, all of which contain $S_{0} \cap S_{0}^{\prime}$. Figure 6 describes the sets $\Omega_{ \pm}$in some detail. Note that although $\Omega_{+}$has a complex shape, the surface integral $\int_{\partial \Omega_{+}}(p+f)|u|^{q-1} \widehat{u} \cdot \mathrm{d} a$ still reduces to $\int_{S \cup S^{\prime}}(p+f)|u|^{q-1} \widehat{u} \cdot \mathrm{d} a$, after negligible contributions from portions of $\partial \Omega$ are omitted. Criterion (4.7) then applies. Now, unlike the case $S_{0} \cap S_{0}^{\prime}=\emptyset$, where $p^{\prime}$ at $O$ remains nonzero in the limit $q \rightarrow \infty, p^{\prime}$ at $O$ in the present case tends to zero. Hence, criterion (4.7) holds for a broader class of shape function $\theta(s)$ than in the non-intersecting case. This makes sense since high-velocity fluid particles on $S_{0} \cap S_{0}^{\prime}$ do not accelerate.

\section{Conclusion}

We have studied the driving term in the equation governing the evolution of $\|u\|_{L^{q}}$, for $q \geq 3$. As is well known, viscous effects can a priori neutralise the contribution to this term from almost everywhere, except for a "pre-singular" set $\Omega$ (a neighbourhood of peak velocity), whose measure diminishes as either $q$ or $\|u\|_{L^{q}}$ (or both) increases. The reduced driving term, expressible as a volume integral over $\Omega$, has been written in a new form amenable to estimation by simple methods. In general, $\Omega$ consists of subregions $\Omega_{+}$and $\Omega_{-}$with positive and negative contributions, respectively, to the driving term. When the 
latter is absent or negligible, straightforward calculations have shown that the driving term is fully suppressed by the dissipation term. On the other hand, when the contribution from $\Omega_{-}$is significant, the problem becomes more involved. This is rather surprising as one may intuitively expect some partial cancellation in the driving term would make it weaker. Nonetheless, a delineation of the subsets $\Omega_{ \pm}$and simple mathematical tricks have turned the driving term into a surface integral over $\Omega_{+}$, whose integrand is sensitive to the shape of $|u|$ in the vicinity of its peak. A regularity criterion depending mainly on this profile has been derived and discussed. We have argued that for a wide range of velocity profiles, viscous effects are strong enough to control the pressure force. However, it is not known whether these profiles are typical of general Navier-Stokes flows.

Turbulence as described by the classical theory is a sea of highly chaotic vortex filaments (possibly decorated by coherent vortices). Presumably, these small-scale objects have large vorticity but moderate velocity and are the products of the direct energy cascade. No theories of turbulence seem to be concerned with the velocity itself, which can be said to be a large-scale quantity. Because of moderate velocity, the classical picture of turbulence is regular by default. Indeed, the theory premise of direct energy cascade, which is arrested at the viscous dissipation scale, is an implicit assumption of regular dynamics. Given such a cascade, the regularity criterion (18)

$$
\|u\|_{L^{6}} \leq c\|\Delta u\|_{L^{2}}^{1 / 3},
$$

can be highly excessive as the bi-vorticity on the right-hand side is super large while the velocity on the left-hand side is moderate. Hence it is infeasible to relate NavierStokes singularity (if realizable) to turbulence in a meaningful manner. One may, however, postulate that turbulence corresponds to a broad class of initial data, while Navier-Stokes singularity occurs for some class of data that evolve in a highly organised manner and resist the energy cascade. In the present analysis, the pre-singular set $\Omega$ and its constituents can be regarded as regions of highly organised and large-scale dynamics. They are thus suitable regions for probes of singularity/regularity.

Acknowledgement. C.V.T. gratefully acknowledges support from the Institute of Mathematics and its Application for a collaborative visit to Alberta. X.Y. is supported by an NSERC Discovery grant. We thank the editor and the anonymous referees for valuable comments.

\section{References}

1. Berselli L. C. and Galdi G. P., Regularity criteria involving the pressure for the weak solutions to the Navier-Stokes equations, Proc. Am. Math. Soc. 130 (2002) 3585-3595.

2. Bosia S., Pata V., and Robinson J. C., A weak- $L^{p}$ Prodi-Serrin type regularity criterion for the Navier-Stokes equations, J. Math. Fluid Mech. 16 (2014) 721-725.

3. Cao C. and Titi E. S., Global regularity criterion for the 3D Navier-Stokes equations involving one entry of the velocity gradient tensor, Arch. Rational Mech. Anal. 202 (2011) 919-932.

4. Chae D. and Lee J., Regularity criterion in terms of pressure for the Navier-Stokes equations, Nonlinear Anal.: Theory, Methods Appl. 45 (2001) 727-735.

5. Constantin P. and Fefferman C., Direction of vorticity and the problem of global regularity for the Navier-Stokes equations, Indiana Univ. Math. J. 42 (1993) 775-789. 
6. Escauriaza L., Seregin G. A., and Sverák V., $L_{3, \infty}$-solutions of Navier-Stokes equations and backward uniqueness, Uspekhi Mat. Nauk. 58 (2003) 3-44.

7. Fan J., Jiang S., Nakaruma G., and Zhou Y., Logarithmically improved regularity criteria for the Navier-Stokes and MHD equations, J. Math. Fluid Mech. 13 (2011) $557-571$.

8. Fan J., Jiang S., and Ni G., On regularity criteria for the n-dimensional Navier-Stokes equations in terms of pressure, J. Differential Eqns 244 (2008) 2963-2979.

9. Gibbon J.D., Weak and strong solutions of the 3D Navier-Stokes equations and their relation to a chessboard of convergent inverse length scales, J. Nonlinear Sci. 29(1) (2019) 215-228.

10. Grujíc Z., A geometric measure-type regularity criterion for solutions to the 3D NavierStokes equations, Nonlinearity 26 (2013) 289-296.

11. Guo Z. and Gala S., Remarks on logarithmical regularity criteria for the Navier-Stokes equations, J. Math. Phys. 52 (2011) 063503.

12. Katz N. and Pavlovic N., A cheap Caffarrelli-Kohn-Nirenberg inequality for the NavierStokes equations with hyper-dissipation, Geom. Funct. Anal. 12 (2002) 355-379.

13. Leray J., Sur le mouvement d'un liquide visqueux emplissant l'espace, Acta. Math. 63 (1934) 193-248.

14. Prodi G., Un teorema di unicità per le equazioni di Navier-Stokes, Ann. Math. Pura Appl. 4 (1959) 173-182.

15. Serrin J., On the interior regularity of weak solutions of the Navier-Stokes equations, Arch. Rational Mech. Anal. 9 (1962) 187-195.

16. Sohr H., A regularity class for the Navier-Stokes equations in Lorentz spaces, J. Evol. Equ. 1 (2001) 441-467.

17. Tao T., Global regularity for a logarithmically supercritical hyperdissipative NavierStokes equation, Anal. Partial Differential Eqns 2 (2009) 361-366.

18. Tran C. V. and Yu X., Pressure moderation and effective pressure in Navier-Stokes flows, Nonlinearity 29 (2016) 2990-3005.

19. Tran C. V. and Yu X., Notes on Prodi-Serrin-Ladyzhenskaya type regularity criteria for the Navier-Stokes equations, J. Math. Phys. 58 (2017) 011501.

20. Tran C. V. and Yu X., Regularity of Navier-Stokes flows with bounds for the velocity gradient along streamline and an effective pressure, London Math. Soc. Lec. Note Ser. 452, Eds. Fefferman C L, Robinson J C and Rodrigo J L, (Cambridge University Press 2018) 249-264.

21. Zhou Y., A new regularity criterion for the Navier-Stokes equations in terms of the gradient of one velocity component, Methods Appl. Anal. 9 (2002) 563-578.

22. Zhou Y. and Lei Z., Logarithmically improved regularity criteria for Euler and NavierStokes equations, Commun. Pure Appl. Anal. 12 (2013) 2715-2719.

23. Ziemer W. P., Weakly Differentiable Functions: Sobolev Spaces and Functions of Bounded Variation, (Springer-Verlag 1989). 